\title{
A Simulator for Drones and FANET management supporting multimedia traffic under human mobility
}

\author{
M. Tropea ${ }^{1}$, F. De Rango ${ }^{1}$ and P. Fazio ${ }^{1}$ \\ ${ }^{1}$ Dimes Department, University of Calabria, via P. Bucci 39/c, 87036 Rende (CS), Italy \\ \{mtropea, derango,pfazio\}@dimes.unical.it
}

Keywords: $\quad$ FANET, Coverage, Drones, Mobility Model, Footprint, Simulator

Abstract: $\quad$ In this paper a simulator for the management of a team of Unmanned Aerial Vehicles (UAVs) and drones has been proposed. This new network is known as Fly Ad-Hoc Network (FANET), and it is a particular type of Mobile Ad-Hoc Network (MANET) but with some specific aspects that allow to provide new services in future generation networks. One of the possible applications is emergency situations or scenario where drones can provide an additional or complementary access networks supporting web services and multimedia traffic. In this paper a simulator for FANET deploying has been proposed providing the possibility to simulate different scenarios with different coverage areas. New coverage model has been included in the features and also interesting human mobility model to support more realistic users mobility. Moreover, additional modules for traffic pattern generation have been implemented to create scenario where mobile users can activate multimedia calls and traffic on FANET. Some simulations have been led out to show how the simulator works.

\section{INTRODUCTION}

In this paper the capability of Unmanned Aerial Vehicles (UAVs) of providing wireless connectivity to users in a coverage area is analyzed. These devices are able to form temporary communication network in order to cover specific regions in many different situations such as disaster and emergency events. In particular, in this paper the task is that to provide a study on a simulation environment created ad hoc for the UAVs/drones coverage purpose. The proposed simulator is realized in java and it is a platform able to permit of simulating different scenarios and situations in which users need to communicate each other. The simulator is able to consider a real map get by Google on which insert a series of specific Points of Interest (PoI) that represent the points in which users move in order to reach the prefixed destination. The simulator permits to set a series of parameters such as users mobility, drone height and coverage radius, different types and percentage of users in the map, type of multimedia traffic and its percentage in the simulation. Moreover, the paper proposes two models, a human mobility model, that tries to provide a way the users use for moving in the covered area, and a footprint model, able to model the drone channel and to provide the mathematical formulation for calculating the correct height of the drone on the base of the coverage radius. Moreover, in order to calculate the available bandwidth for the drones in the path followed by the users in their movements, a standard Link State protocol has been considered in which the topological changes are due to change in the parameters status (bandwidth along the path) and the protocol update are operated on periodical base. The results of the simulative campaigns are shown in order to validate the goodness of the proposed simulator software. There are other important topics that are object of research about Drones beyond the coverage providing such as that regards the bandwidth management performing mobility prediction of the users and the opportune admission call (Fazio et al., 2012; Fazio et al., 2016; Fazio et al., 2017). The rest of this paper is organized as follows: Section 2 presents the related work on the considered research topic. In Section 3, a description of the FANET simulator is given. In Section 4 , we describe the simulation environment created in java. Section 5 shows the simulator implementation details. The numerical results are presented in Section 6. Finally, Section 7 concludes the paper.

\section{RELATED WORK}

In the last years a lot of research has been focused on the Unmanned Aerial Vehicles (UAVs) technology. 
Many researchers have analyzed and studied many different aspects of these new devices in order to show their potentiality and capacity in many real contexts.

\subsection{Simulator of Drones and UAVs}

In this paragraph some of the literature works about simulators proposed for UAVs/Drones devices under different points of view and applicative domains will be presented. The papers (De Rango et al., 2017a; De Rango et al., 2017b) propose a simulator suitable for the agriculture domain in order to design novel coordination and control techniques of a UAVs team. The works presented many coordination techniques both for monitoring the area and for coordinating the actions of the drones in the presence of parasites in order to analyze how the performance can significantly change if more constraints, such as energy, communication range, resource capacities, are accounted. In (Bustamante et al., 2017) the authors present the development of a virtual reality simulator for the management of a UAV (Unmanned Aerial Vehicle), focused on improving the quality of grownup people. This research has collected characteristics of gestures and physical movements from users made by other related research in order to study the same interaction within a virtual world. In (Lombardo et al., 2016) the authors adopt the popular approach of leveraging the X-Plane flight simulator to simulate the environment and aircraft dynamics. This approach has found recent success in visualizing and optimizing algorithms for basic UAV flight control as well as formation flight. In (Zema et al., 2017) the authors describe a novel simulation architecture to implement distributed networked control systems. Their proposal consists in the integration of alreadypresent and validated solutions into a compact package that features scalability and negligible architectural delays.

\subsection{Coverage issues in Drones}

In this paragraph some works about coverage issues are shown. This typology of research is very studied and analyzed because the FANET represents a technology able to improve and guarantee prompt help in many real emergency situations. Then, many aspects of the coverage and connectivity issues are object of study. In (Al-Hourani et al., 2014) the authors provided a statistical generic Air-to-Ground RF propagation model for Low Altitude Platforms (LAPs), that can substantially facilitate the planning efforts of airborne wireless services, since the RF planning can be performed based on merely simple urban parameters, rather than depending on site specific 3D-models that are unlikely to be easily available and updated. In (Park et al., 2015), an effective coverage decision algorithm is proposed, which aims at providing seamless handover and thus establishing a fully connected aerial network. They further calculate the seamless handover success probability and the false handover initiation probability to evaluate the proposed coverage decision algorithm. (Sae et al., 2016) studies the coverage aspects of a Low Altitude Platform (LAP) system that can form a temporary communication network. The system consists of multiple autonomous drones equipped with dual-band Wi-Fi access points (APs) with ad hoc capabilities to form a mesh network. The suitability of the LAP system is evaluated from the coverage point of view with calculations and simulations. In (Xie et al., 2017), the authors intend to study the optimal deployment density of Drone Small Cells (DSCs) to achieve maximum coverage considering the inter-cell interference considering both LoS and NLoS links.

\section{FANET SIMULATOR}

The simulator to be implemented must allow to analyze the trend over the time of a network composed of drones. The drones fly over a city and communicate with each other trying to create a network through an appropriate messages exchange. During the flight the drones will have to be able to guarantee a connection to the people who are within their coverage range and who request the connection. The purpose of the simulations is therefore to analyze the network in terms of bandwidth occupation, delays and lost packet. The simulation area is a map that can be taken by google maps platform in order to make the proposed environment extremely general and parametric. This permits of considering whatever area in which we want analyze the human movements on the basis of chosen area characteristics while the users utilize their multimedia applications such as Video and VoIP calls. The chosen area has been represented by the class "Grid". It contains all the information as: number of people in the map; the number of clients classified by typology; number of used drones; size of the field; list of Points of Interest where a person can stop.

While the main actors are represented by the classes:

- Drone: could be defined as the main class, as guarantor of the functioning of each protocol that is developed, such as the Link State, for the realization of the network. 


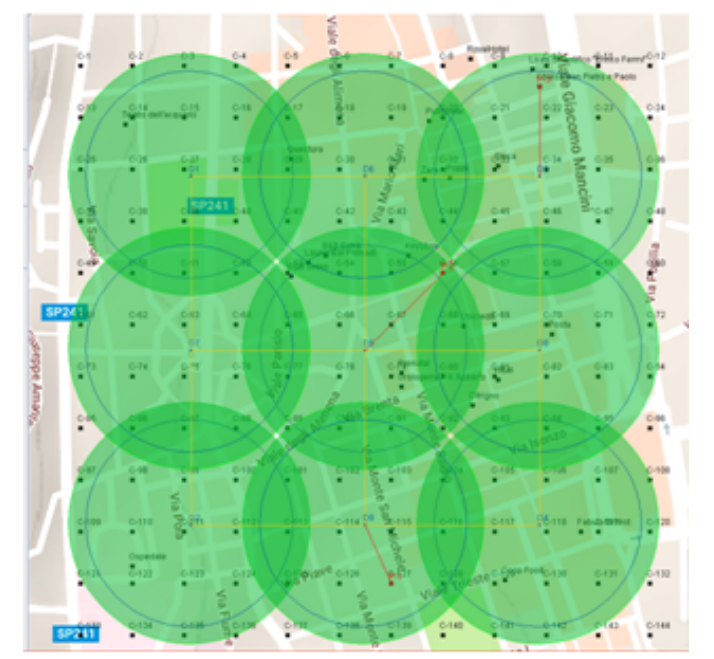

Figure 1: Reference Scenario with drone coverage footprint.

- Client: a person must follow a specific model of mobility, which we will see in the following, and must be able to have the possibility of making audio and video calls. He must also be able to send information, such as his position, to the people who request it.

As said so far, the paper focuses on the realization of a simulator that allows to analyze a network composed of drones, a FANET. For the realization of this network we have relied on a classical graph structure, in which the nodes represent the Drones and the Clients, and the links represent the connections between them. The graph is created considering a sparse graph since the number of edge departing from a node is independent of the number of nodes in the graph. The network will be created by exchanging messages between nodes that are at a distance allowing communication. So the number of links varies according to the number of nodes in the network and to their distance. A key feature of our simulations is to keep track of changes in link bandwidth, so, in addition to information regarding the distance between nodes, an edge stores the bandwidth parameter. Furthermore, an edge is always identified by the couple of nodes that it links. To manage the neighborhood information between nodes, a list is created for each vertex where neighboring vertices will be stored.

\subsection{FANET creation and management}

The network is created dynamically by exchanging messages between drones and also between drones and clients. The main feature of the network is that it must be able to create and manage itself autonomously even when there are topological changes or changes that affect the status of the links. For this task, in this work, the FANET network uses one of the most popular routing protocols, the Link State protocol. The method "StartLinkState()" was implemented in the Drone class to manage Link State operation. This method is called up whenever a change of state occurs in the network.

The following messages are sent for the creation of the FANET:

- HELLO: it allows establishing the connection between drones and between drone and client;

- $A C K$ : drone message of response to the received Hello message;

- BYE: it allows the disconnection from the drone, both for drones and for clients;

- LSA (Link State Advertisement): it contains the Link State Table of the reference drone and will be forwarded in flooding to all the nodes of the network.

- LSU (Link State Update): it is a Link Update (LU) collector and allows network traffic reduction.

- $L U$ : it is not a proper packet of the standard Link State operation. It is a message that the drone sends itself whenever there is a band update on one of its links. To reduce traffic, these messages are grouped in a single LSU message that will be manage every 30 seconds. The drone keeps track of the status changes of its links and at the end it will update its LSA Database and generate an LSA packet that will contain its own table of neighbor nodes with updated bandwidth capabilities.

All these packets inherit from the general class "LinkStatePacket", which contains the standard information of each packet and it will be characterized by:

- the $I D$ of the node that generates them;

- a sequence number that will be increased for each generation;

- a numeric value to represent the Time To Live (TTL) of the packet.

In the simulation process, each created drone will have two tables that will continue to update during the simulation: 1) LSA Table: it keeps track of neighboring nodes. This table will be the one that is sent from time to time for topological changes. In fact, if the drone realizes that some neighboring node is no longer connected, or notices a change in the status of the links, then it will put the related changes in this table and forward it to the neighboring nodes via an LSA message. 2) $L S A D B$ : it keeps track of 
all the information on the network and will be created and modified each time an LSA from the other nodes on the network is received. When the simulator starts, each drone must send a Hello message to the nearby drones. The neighboring drones are all those drones that are within a certain range of coverage, set to 300 meters. The neighboring drones will respond and, upon receiving the $A C K$, the drone that made the Hello request will create a new line within own LSA $D B$ and own LSA Table.

Once all the $A C K$ have been received, the drones generate a $L S A$ packet which will be forwarded to all the neighboring nodes, which process the data in the table contained in the packet and update their LSA Database. They will then forward the received LSA packet to all the neighbors, except to the node from which they received it. Obviously, in this way, it will be possible to obtain equal $L S A s$ from several drones and, in fact, a check on the sequence number of the received LSA packet is always performed. Each drone stores a list consisting of couple of values that will indicate: the $I D$ of the nodes from which an LSA was received; the highest sequence number relative to the LSAs received from that specific node. If an LSA is received with a sequence number lower than that stored in the list then it will be discarded and it will not managed. The LSA packets will then be sent in flooding in the network whenever there is a topological change, as can be the connection/disconnection of a new node and in the simulator this happens whenever a Client connects or disconnects from the network.

\subsection{Human Mobility: Mobility Model in Urban Space}

There are a lot of studies on this subject and several interesting models for our case, including a study by the University of Milan (Jahromi, 2017). They analyzed data sets on smartphone call information in Milan for 67 days, detecting over 69 million phone calls and over 20 million text messages. Later, they also analyzed the WiFi datasets and GPS data with which they found the movement of 178 people within 4 years (between 2007 and 2011).

After the analysis of the data, they have obtained a model of people mobility based on the classification in 3 categories of Points of Interest (PoI) that are visited daily:

- Mostly Visited PoI (MVP): the places most visited by a user that may be their workplace, or their home.

- Occasionally Visited PoI (OVP): the places of interest of a user, but occasionally visited. Often it corresponds to favorite places or meeting points visited during the week.

- Exceptionally Visited PoI (EVP): points of interest rarely visited.

On this type of model we developed our idea of mobility which includes 3 types of users:

- Worker: who represents the classic type of person who spends most of the day at work. The students can fit into this category.

- Housewife: who represents the type of person who spends most of the time, especially in the morning, at home.

- Pensioner: who represents the type of person who is free to move.

Each type of user will spend the time in the day in a different way and will visit most likely the points of major interest, also based on time slots, so we tried to represent this mobility in the simulator by considering the following models.

\subsection{Footprint model}

For that concerns air to ground channel model in the considered urban scenario, the ground receiver can receive two main signals: one is the Line of Sight (LoS) signal and the other one is the Non-Line of Sight (NLos) signal (Al-Hourani et al., 2014). These two typologies of signal can be considered as separately conditions and, then, they are studied considering different probabilities of occurrence. These two propagation conditions can be considered separately assuming they have different probabilities of occurrence. Typical, as discussed in (Feng et al., 2006), it can be assumed that the signal received by the destination can be considered only in one of the two previous typologies. Each typology has a specific probability of occurrence which is a function of environment, density and height of buildings and elevation angle. The probability of receiving LoS and strong NLoS components are significantly higher than fading (Feng et al., 2006). Therefore, the impact of small scale fading can be neglected. A common approach to model air to ground propagation channel is to consider LoS and NLoS components along with their occurrence probabilities separately. Note that for NLoS connections due to the shadowing effect and reflection of signals from obstacles, Path Loss $(P L)$ is higher than LoS. Hence, in addition to the free space propagation loss, different excessive path loss values are assigned to LoS and NLoS links. As it is possible to view in figure 2 a coverage area is represented in which $h$ is the height of the drone and $r$ is the radius of 
the coverage, $d$ is the distance between an user on the edge of the coverage area and the drones in the sky. $d$ is given by the following formula: $d=\sqrt{r^{2}+h^{2}}$, instead the angle $\theta$, in radiant, between $r$ and $d$ is given by: $\theta=\tan ^{-1}(h / r)$.

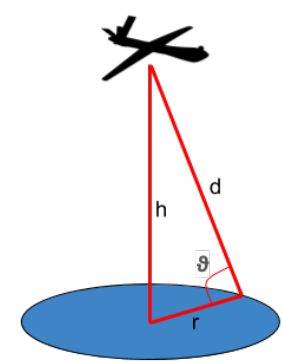

Figure 2: UAV Footprint

On the basis of this consideration, in the following the formulas respectively of $P L$ for the LoS and NLoS conditions as in (Al-Hourani et al., 2014):

$$
\begin{gathered}
P L_{L o S}(d B)=20 \log \left(4 f_{c} d / c\right)+\xi_{L o S} \\
P L_{N L o S}(d B)=20 \log \left(4 f_{c} d / c\right)+\xi_{N L o S}
\end{gathered}
$$

where $P L_{L o S}$ and $P L_{N L O S}$ are the average (PL) for LoS and NLoS links, $\xi_{L o S}$ and $\xi_{N L O S}$ are the average additional loss to the free space propagation loss which depend on the environment, $c$ represents the speed of light, $f_{c}$ the carrier frequency. The probability $P_{r}$ of having LoS connections at an elevation angle of $\theta$ is given by (Zheng et al., 2013)

$$
P_{r}(L o S)=\frac{1}{1+\alpha \cdot \exp (\beta[(180 / \pi) \theta-\alpha)]}
$$

where $\alpha$ and $\beta$ are constant values which depend on the environment (rural, urban, dense urban, etc.). The NLoS probability is $P_{r}(N L o S)=1-P_{r}(L o S)$. The formula represented in equation 3 indicates that the probability of having LoS connection between the drone in the sky as Access Point (AP) and the users in the coverage area is an increasing function of $\theta$. This means that by increasing the elevation angle $\theta$, the shadowing effect decreases and clear LoS path exists with high probability. Finally, the average $P L$ as a function of the altitude $h$ and coverage radius $r$ becomes:

$$
\overline{P L}(r, h)=P_{r}(L o S) \cdot P L_{L o S}+P_{r}(N L o S) \cdot P L_{N L o S} .
$$

On the base of the previous drone channel model we want to provide a formula for computing the optimal altitude $h$ for the maximum ground coverage area
Table 1: Considered parameters in mathematical formulation

\begin{tabular}{|c|c|}
\hline Parameter & Value \\
\hline$f_{c}$ & $2 \mathrm{GHz}$ \\
\hline$\xi_{\text {LoS }}$ & $1 \mathrm{~dB}$ \\
\hline$\xi_{N L O S}$ & $20 \mathrm{~dB}$ \\
\hline$\alpha$ & 9.6 \\
\hline$\beta$ & 0.28 \\
\hline$\gamma_{t h}$ & $10 \mathrm{~dB}$ \\
\hline
\end{tabular}

of radius $r$. Consider a drone in the sky able to transmit a signal of power $P_{\text {tran }}$, then the received power $P_{\text {rec }}$ can be represented by the following formula:

$$
P_{\text {rec }}(d B)=P_{\text {tran }}-P L(r, h) .
$$

Once the received power $P_{\text {rec }}$ is calculated, an user in the coverage area of the drone at an height of $h$ is able to receive the signal if its Signal to Noise Ratio (SNR) is greater than a fixed threshold $\left(\gamma_{t h}\right)$. This means that:

$$
\gamma(r, h)=\frac{P_{r e c}}{N}>\gamma_{t h}
$$

where $N$ is the noise power. From the previous formula, it is possible to assert that to find the maximum achievable coverage radius we should have: $\gamma(r, h)=\gamma_{t h}$. For a fixed transmit power, the optimal drone height which results in maximum coverage is computed by solving the following equation as it is possible to view in (Feng et al., 2006):

$$
\frac{180\left(\xi_{N L o S}-\xi_{L o S}\right) \beta Z}{\pi(Z+1)^{2}}-\frac{20 \mu}{\log (10)}=0
$$

where $Z=\alpha \cdot \exp \left(\left[(180 / \pi) \tan ^{-1}(\mu)-\alpha\right]\right)$ and $\mu=h / r$. By solving equation $7, \mu_{o p t}=h_{o p t} / r_{\max }$ is computed and using equation $5, h_{o p t}$ and $r_{\max }$ are found.

\section{SIMULATION ENVIRONMENT}

In order to allow the movement of people, an urban scenario was created taking as an example the google map of the center of the city of Cosenza, in the southern of Italy. Some Points of Interest (PoI) have been chosen, such as shops, restaurants, hotels, churches, etc. and then people's homes: a grid of 144 houses was chosen to cover most of the map. Subsequently, the people movement has been realized. Each person will start its day at 00:00 and will stay at home until $8 \mathrm{am}$. Later, based on the proposed model, people will move throughout the day. In the simulator it is possible to set, in addition to the number of people, the percentage of workers, housewives 
and pensioners. The movements of the people are determined by the "start()" method, which based on the time slots and the various probabilities determines the next action of the person and, in particular, the next destination. Once the next destination is determined, each person will try to reach it by following a path. The proposed algorithm for finding the best route consists in searching each time for a next PoI that can be reached within a radius of 90 meters and that represents the PoI closer to the destination. To better understand the reasoning, it is possible to take the following figure, figure 3, as an example: a person moves from point $S$ to point $D$. Within his range of action (where there are points 1, 2 and 3, as it is possible to view in figure) the point 2 satisfies the conditions of the algorithm, that is, it represents the point reachable from $S$ within a radius of 90 meters and closer to the destination.
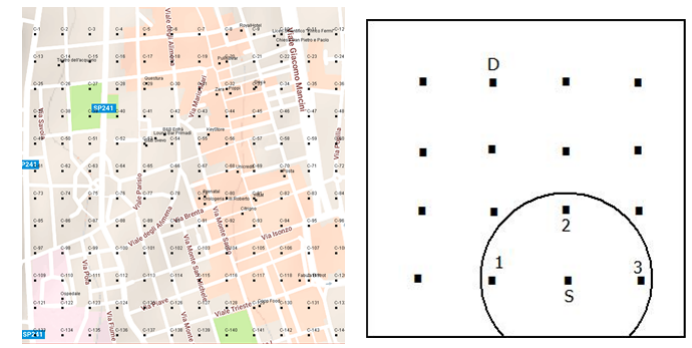

Figure 3: Example of search for the next PoI to reach the destination

A grid arrangement was then chosen, therefore, to avoid the problem of not finding another PoI within the radius of action that would allow the movement. A person performs his movements towards the chosen destination, moving at a speed of $3 \mathrm{~km} / \mathrm{h}$. There is a procedure that is the heart of the whole movement as it recursively, if after a movement the destination has not been reached, recalculates the next PoI generating again the events for the movement. Usually, a person who is not working or not at home can stay in a PoI with a time varying between 10 and 60 minutes each time. All activities end at 22:00, the limit beyond which the day of the people is considered concluded. People will return home and this determines the end of the simulation.

\section{SIMULATOR IMPLEMENTATION}

We have implemented a simulator in order to analyze various aspects of the FANET. In particular, the goal is to control how the network behaves during a day using a Link State Standard approach in

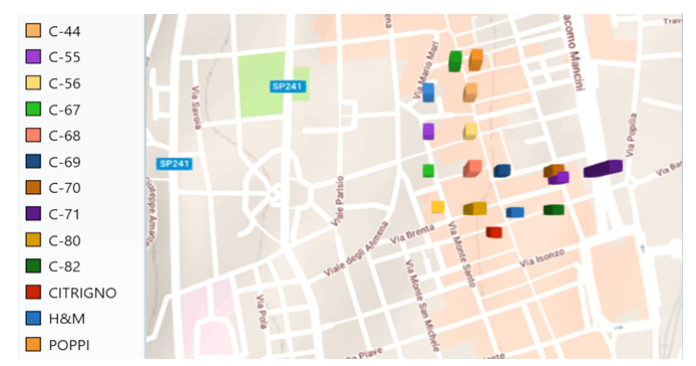

Figure 4: View of the PoI visited by a person during a day

which network drones will send an LSA to neighboring nodes whenever a topological change occurs. We have considered as simulation area a portion of the city of Cosenza (in the southern of Italy). In particular, we have considered the surrounding area of "Corso Mazzini" (one of the most important street of the city) where the people who move in this area are able to make audio or video calls. In our work we have considered three different video and audio calls distributions as shown in the following:

- $30 \%$ of audio streams and $70 \%$ of video streams;

- $50 \%$ of audio streams and $50 \%$ of video streams;

- $70 \%$ of audio streams and $30 \%$ of video streams.

Simulations were carried out considering the number of users in the area of interest in increasing. In addition to the variable parameters, different fixed parameters have been set for the simulations: we have set the drones without problems of autonomy; each drone will have available $10 \mathrm{Mbps}$ of bandwidth dedicated to the call admission of users within its range of coverage; the range of coverage has been set with a radius of $175 \mathrm{~m}$ and the optimal height of the drone has been obtained equal to about 120 meters from the ground (Hayajneh et al., 2016; Mozaffari et al., 2015); all drones are equipped with a buffer that stores video and audio packets before forwarding them to the network. The size of this buffer has been fixed to a maximum of 50 packets; the communication range between the drones has been set to 300 meters; the movement speed of people has been set at $3 \mathrm{Km} / \mathrm{h}$; a person can move from one PoI to another within a radius of 90m; 166 PoIs were chosen: 144 houses placed as checkerboard and 22 other PoIs including shops, refreshment points and entertainment; each person will be able to make a call each time it is in a PoI. Considering the proposed mobility model, it can therefore be noted that, with $70 \%$ of workers spending most of their time at work, the flow of calls in the network will be quite limited; to have a more realistic case we have chosen to have users divided as follows: $70 \%$ of workers; $20 \%$ of housewives; $10 \%$ of pensioners. For all the simulations, a network topology 
consisting of 9 drones, distant 252 meter each other, in rows of 3 has been considered, able to cover most of the area of interest. The resultant network will be so composed as shown in fig 1.

\section{SIMULATION RESULTS}

A series of simulations have been started with a growing number of people. The network was analyzed from a minimum of 50 people to a maximum of 150. For each simulation the considered parameters have been:

- jitter of audio and video streams;

- delay of audio and video streams;

- number of packets sent, received and lost;

- bandwidth requests accepted and refused;

- number of calls made;

- percentage of bandwidth occupied for each drone for that concerns the admission of calls.

The simulation results show the trend of some parameters such as sent and received packets, accepted and refused requests of bandwidth, delay of the two typologies of streaming. These parameters allow to evaluate the goodness of the proposed simulator in which we have varied some ingress parameters. In particular, in figures 5 it is possible to view the number of sent and received packets while the number of accepted bandwidth requests, calls done and refused requests are shown in figure 6 . The figure 5 shows how the number of packets increases varying the number of people and how the number of lost packets is very low. From the figure 6 it can be seen how the number of bandwidth requests increases with the number of people and how, therefore, the number of calls made and the number of bandwidth requests refused will increase. In addition, in the figure 7 , we see which drones have a greater demand load and, therefore, a higher percentage of occupied bandwidth. The drones 6 and 8 are those with the highest occupancy rate, due to a greater concentration of PoIs in the area covered by them. Furthermore, it can be seen how the percentage of bandwidth increases for an increasing number of people. In the figures 8 and 9 it is possible to view the delays in both multimedia considered traffics. Figure 8 shows the delay in user communications that consider VoIP multimedia traffic. Finally, figure 9 shows the delays, minimum, medium and maximum for video traffic. The figures show delays in line with the typical delay of these typologies of multimedia traffics.

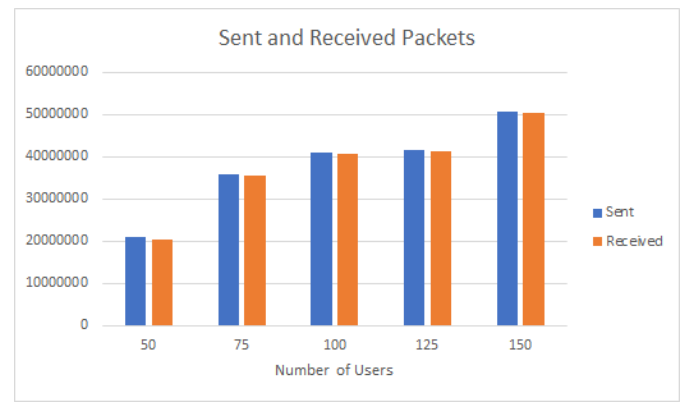

Figure 5: Sent and Received packets vs number of users

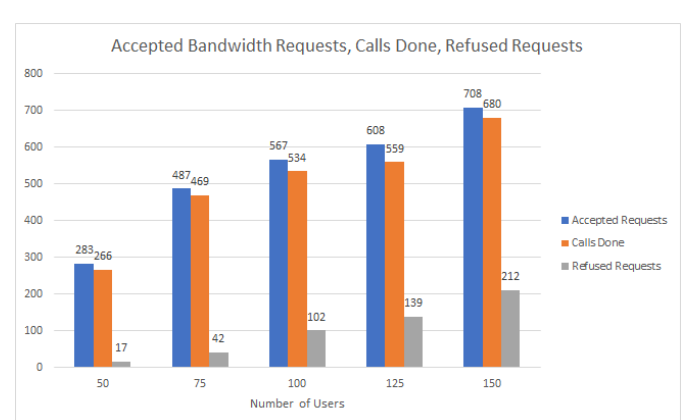

Figure 6: Accepted bandwidth requestes, Calls done, refused requestes vs number of users

\section{CONCLUSIONS}

In this paper we present the implementation of a UAVs/drones simulator environment created in java and useful for analyzing different aspects of the so called FANET. The possibility of providing temporary communication network composed of these new kinds of devices is a very important research topic of these last years. This aspect is very important in many cases, first of all in emergency situations when it is important to guarantee efficient communications in the considered area. In this paper we propose a simulation environment for UAVs coverage. The proposed simulator is created in order to provide a platform able to permit of simulating different scenarios and

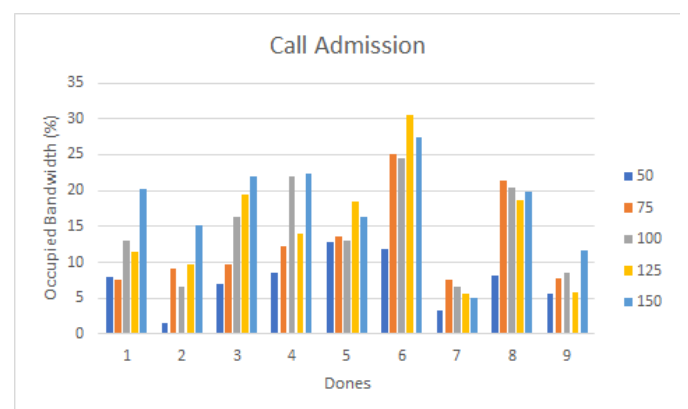

Figure 7: Number of call admission vs drone for different number of users 


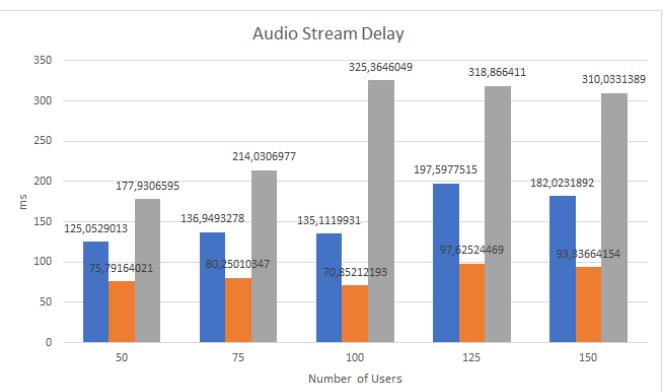

Figure 8: Audio stream delay (min, medium, max) vs number of users

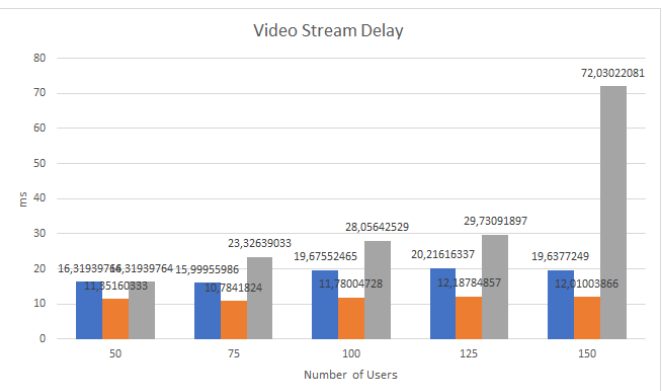

Figure 9: Video stream delay (min, medium, max) vs number of users

cases in which users need to communicate each other. The performance evaluation of our proposed simulator has been considered using multimedia traffic such as video and audio streaming as typology of users' applications. We have simulated a team of drones able to cover a specif area where the considered footprint and human mobility model have been taken in consideration for carried out simulation results.

\section{REFERENCES}

Al-Hourani, A., Kandeepan, S., and Jamalipour, A. (2014). Modeling air-to-ground path loss for low altitude platforms in urban environments. In 2014 IEEE Global Communications Conference, pages 2898-2904.

Bustamante, A., Guerrero, G., Rodrigues, N., and Pereira, A. (2017). Uav simulator for grown-up people quality of life enhancement. In 2017 12th Iberian Conference on Information Systems and Technologies (CISTI), pages 1-6. IEEE.

De Rango, F., Palmieri, N., Santamaria, A. F., and Potrino, G. (2017a). A simulator for uavs management in agriculture domain. In 2017 International Symposium on Performance Evaluation of Computer and Telecommunication Systems (SPECTS), pages 1-8. IEEE.

De Rango, F., Palmieri, N., Tropea, M., and Potrino, G. (2017b). Uavs team and its application in agriculture: A simulation environment. SIMULTECH, 2017:374379 .
Fazio, P., De Rango, F., and Tropea, M. (2017). Prediction and qos enhancement in new generation cellular networks with mobile hosts: A survey on different protocols and conventional/unconventional approaches. IEEE Communications Surveys \& Tutorials, 19(3):1822-1841.

Fazio, P., Tropea, M., De Rango, F., and Voznak, M. (2016). Pattern prediction and passive bandwidth management for hand-over optimization in qos cellular networks with vehicular mobility. IEEE Transactions on Mobile Computing, 15(11):2809-2824.

Fazio, P., Tropea, M., Veltri, F., and Marano, S. (2012). A novel rate adaptation scheme for dynamic bandwidth management in wireless networks. In 2012 IEEE 75th Vehicular Technology Conference (VTC Spring), pages $1-5$.

Feng, Q., McGeehan, J., Tameh, E. K., and Nix, A. R. (2006). Path loss models for air-to-ground radio channels in urban environments. In 2006 IEEE 63rd Vehicular Technology Conference, pages 2901-2905.

Hayajneh, A. M., Zaidi, S. A. R., McLernon, D. C., and Ghogho, M. (2016). Optimal dimensioning and performance analysis of drone-based wireless communications. In 2016 IEEE Globecom Workshops (GC Wkshps), pages 1-6. IEEE.

Jahromi, K. K. (2016/2017). Human mobility in urban space. Doctoral Dissertation, Universitas Studiorum Mediolanensis.

Lombardo, C., Miller, I., and Wallace, J. (2016). Studying the interaction of uas and human pilots using the $\mathrm{x}$ plane flight simulator. In 2016 International Conference on Unmanned Aircraft Systems (ICUAS), pages 557-561. IEEE.

Mozaffari, M., Saad, W., Bennis, M., and Debbah, M. (2015). Drone small cells in the clouds: Design, deployment and performance analysis. In 2015 IEEE Global Communications Conference (GLOBECOM), pages 1-6. IEEE.

Park, K., Cho, B., Park, K., and Kim, H. (2015). Optimal coverage control for net-drone handover. In 2015 Seventh International Conference on Ubiquitous and Future Networks, pages 97-99.

Sae, J., Yunas, S. F., and Lempiainen, J. (2016). Coverage aspects of temporary lap network. In 2016 12th Annual Conference on Wireless On-demand Network Systems and Services (WONS), pages 1-4.

Xie, J., Dong, C., Li, A., Wang, H., and Wang, W. (2017). Optimal deployment density for maximum coverage of drone small cells. In 2017 IEEE 86th Vehicular Technology Conference (VTC-Fall), pages 1-6.

Zema, N. R., Trotta, A., Sanahuja, G., Natalizio, E., Di Felice, M., and Bononi, L. (2017). Cuscus: Communications-control distributed simulator. In 2017 14th IEEE Annual Consumer Communications \& Networking Conference (CCNC), pages 601-602.

Zheng, Y., Wang, Y., and Meng, F. (2013). Modeling and simulation of pathloss and fading for air-ground link of haps within a network simulator. In 2013 International Conference on Cyber-Enabled Distributed Computing and Knowledge Discovery, pages 421426. 